\title{
Dynamic soft tissue mobilisation increases hamstring flexibility in healthy male subjects
}

\author{
D Hopper, S Deacon, S Das, A Jain, D Riddell, T Hall, K Briffa
}

Br J Sports Med 2005;39:594-598. doi: 10.1136/bjsm.2004.011981

See end of article for authors' affiliations

.....................

Correspondence to: Diana Hopper, Curtin University of Technology, School of Physiotherapy, Shenton Park, WA 6008, Australia; d.hopper@ curtin.edu.au

Accepted

21 December 2004

\begin{abstract}
Objectives: The purpose of this study was to investigate the effect of dynamic soff tissue mobilisation (STM) on hamstring flexibility in healthy male subjects.

Methods: Forty five males volunteered to participate in a randomised, controlled single blind design study. Volunteers were randomised to either control, classic STM, or dynamic STM intervention. The control group was positioned prone for $5 \mathrm{~min}$. The classic STM group received standard STM techniques performed in a neutral prone position for $5 \mathrm{~min}$. The dynamic STM group received all elements of classic STM followed by distal to proximal longitudinal strokes performed during passive, active, and eccentric loading of the hamstring. Only specific areas of tissue tightness were treated during the dynamic phase. Hamstring flexibility was quantified as hip flexion angle (HFA) which was the difference between the total range of straight leg raise and the range of pelvic rotation. Pre- and post-testing was conducted for the subjects in each group. A one-way ANCOVA followed by pairwise post-hoc comparisons was used to determine whether change in HFA differed between groups. The $\alpha$ level was set at 0.05 .

Results: Increase in hamstring flexibility was significantly greater in the dynamic STM group than either the control or classic STM groups with mean (standard deviation) increase in degrees in the HFA measures of $4.7(4.8),-0.04(4.8)$, and $1.3(3.8)$, respectively.

Conclusions: Dynamic soft tissue mobilisation (STM) significantly increased hamstring flexibility in healthy male subjects.
\end{abstract}

$\mathrm{F}$ exibility is considered an essential element of normal biomechanical functioning in sport. ${ }^{1}$ The literature reports a number of associated benefits of flexibility including improved athletic performance, reduced injury risk, prevention or reduction of post-exercise soreness, and improved coordination. ${ }^{1-4}$ Some studies have shown that decreased hamstring flexibility is a risk factor for the development of patella tendinopathy and patellofemoral pain, ${ }^{56}$ hamstring strain injury, ${ }^{6}$ and symptoms of muscle damage following eccentric exercise. ${ }^{7}$

Techniques commonly used by athletes to increase flexibility include static and ballistic stretching as well as proprioceptive neuromuscular facilitation. ${ }^{89}$ Nonetheless, recent literature reviews have indicated that stretching does not provide significant benefits. ${ }^{10}{ }^{11}$ Similarly, despite a lack of supporting evidence, ${ }^{12}$ it is widely believed amongst athletes, coaches, and therapists that massage is an effective treatment modality for increasing flexibility. ${ }^{13}$

The effect of massage on restoring muscle flexibility has not been extensively researched; however, several studies have investigated the use of massage as a treatment option for delayed onset muscle soreness (DOMS). ${ }^{14-19}$ These studies have evaluated the use of massage to prevent strength losses, reduce muscle soreness, and maintain joint range of motion (ROM). Few of these studies have shown positive effects ${ }^{16} 19$ and many contain major methodological flaws, such that a clear picture of the effectiveness of massage on DOMS cannot be generated. ${ }^{15}$ Existing studies on massage describe significant variations in the soft tissue techniques, which makes direct comparisons between these studies difficult. ${ }^{12}$ It is evident that an effective method of influencing flexibility has yet to be identified.

A hierarchical dynamic deep muscle tissue model (DDMT) was developed to treat athletes with muscle tightness and associated soft tissue problems. ${ }^{20}$ This DDMT model consisted of a series of progressions from traditional to dynamic techniques which concentrated on one specific area of muscle tightness. It is hypothesised that incorporating active contractions into a massage protocol may increase muscle perfusion and decrease muscle stiffness. ${ }^{21}$ Clinical experience suggests that the DDMT model is an efficient, pain free intervention that appears to have an immediate effect on improving hamstring flexibility. However, at present, there is no scientific evidence to support this form of intervention for muscle tightness problems. Furthermore, the current literature provides no information on the effectiveness of soft tissue techniques used in conjunction with dynamic functional techniques. Therefore, the purpose of this study was to determine the effect of dynamic STM on hamstring flexibility in healthy male subjects.

\section{METHODS}

\section{Study design}

This study was conducted at the School of Physiotherapy, Curtin University of Technology, Australia, using a randomised, controlled single blind design. Two massage interventions (classic STM and dynamic STM) were compared with a no intervention control. The independent variable was the intervention group (control, classic STM, and dynamic STM interventions) and the dependent variable was the hip flexion angle (HFA).

\section{Subjects}

Forty five healthy male volunteers between the ages of 18 and 35 years, with a mean age (standard deviation) of 23.7 (4.6) years, participated in the study. The inclusion criteria consisted of a straight leg raise (SLR) of between $40^{\circ}$ and $70^{\circ}$, which was measured at the perceived onset of hamstring muscle tightness. Subjects were excluded if they had a

Abbreviations: DDMT, deep muscle tissue model; DOMS, delayed onset muscle soreness; HFA, hip flexion angle; ICC, intraclass correlation; LSD, least significant difference; ROM, range of motion; SLR, straight leg raise; STM, soft tissue mobilisation 
history of a hamstring injury within the last 2 years, low back pain in the last 2 months, or any indication of lumbar or lower limb neurological compromise. The Curtin University of Technology Human Research Ethics Committee approved this study and all subjects signed an informed consent form.

\section{Intervention protocols}

Using SPSS Version 10.0, an automatically generated random numbers table was used to randomly assign participants to either the control, classic STM, or dynamic STM intervention.

\section{Control}

In the control group, subjects were positioned in a prone lying position for a period of $5 \mathrm{~min}$.

\section{Classic STM intervention}

For the classic intervention, all subjects were positioned lying prone with the hip and knee in a neutral relaxed position. Each subject received a massage, based on traditional Swedish massage techniques, on the hamstring muscle group. The specific techniques included effleurage, kneading, picking up, and shaking. ${ }^{22}$ The total massage consisted of five strokes of each of these techniques and was completed in 5 min.

\section{Dynamic intervention}

Before the dynamic intervention was implemented, the subject received the same classic massage techniques within the same time frame of approximately $5 \mathrm{~min}$. However, the dynamic intervention was divided into hierarchical progressions which involved assessment and identification of a specific area of hamstring tightness, the application of three different dynamic techniques, and reassessment after each of these techniques.

To assess the hamstring muscle group, the subject remained in the prone position and deep longitudinal strokes were applied to this entire muscle group. Once the specific area of hamstring muscle tightness was located, the remaining treatment was limited to this target area (fig l).

To execute the dynamic intervention, the subject was moved into a supine position with the hip and knee flexed to $90^{\circ}$. In this position, all dynamic techniques worked the hamstring muscle length from three quarter to end ROM.

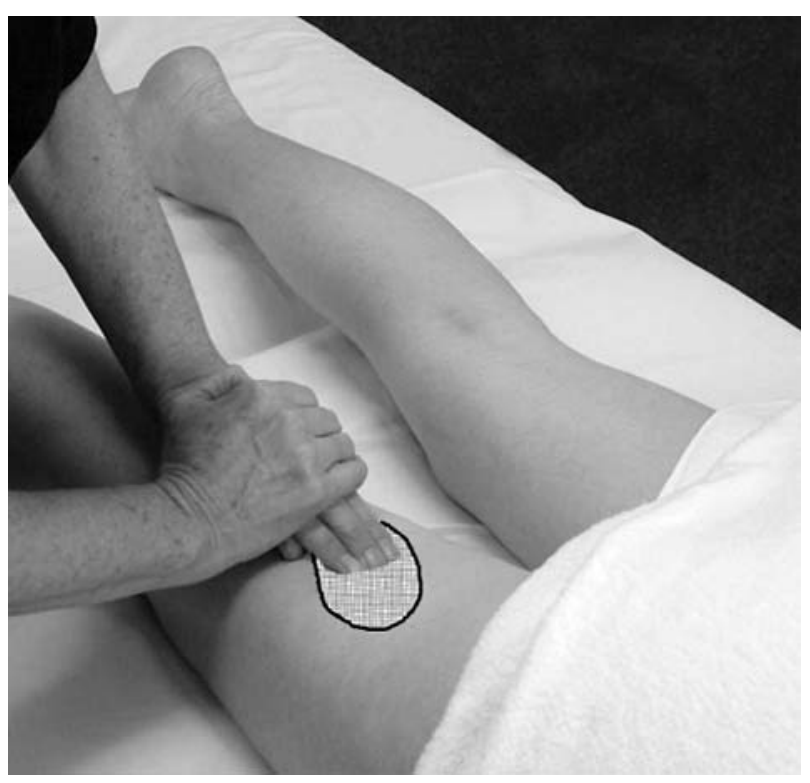

Figure 1 Identification of the area of muscle tightness using longitudinal strokes. (Photograph reproduced with consent)
Deep longitudinal strokes were applied in a distal to proximal direction to the area of hamstring tightness when the leg was passively moved to the hamstring lengthened position (fig 2). Five strokes were applied and $20 \mathrm{~s}$ of shaking was performed at the completion of this technique. The specific area of hamstring tightness was reassessed to determine whether the surface area of the site of muscle tightness was reduced. If this reduction had occurred, then the next progressive dynamic technique was applied. However, if this area of muscle tightness was not reduced, then treatment was stopped. This criterion was applied for all dynamic techniques.

The same sequence was implemented for the next dynamic technique. During this technique, the subject was required to actively extend their leg, in order to achieve reciprocal inhibition of the hamstrings. In the final technique, the subject was required to work the hamstring muscle group eccentrically by creating tension in the therapist's hand as the muscle was elongated to the end ROM. During this movement, the therapist performed five deep distal to proximal longitudinal strokes over the reduced hamstring area of muscle tightness. It was found that a limited number of these strokes reduced the possibility of delayed onset of muscle soreness.

In this study, all subjects proceeded through the dynamic STM progressions and no subjects complained of pain. However, on occasion, subjects reported a feeling of deep pressure during the eccentric loading phase of these techniques.

Overall, the time to complete the combination of both the classic and the dynamic interventions was approximately $8 \mathrm{~min}$.

\section{Testing procedure}

Upon arrival, subjects were assessed for suitability based on the inclusion and exclusion criteria. Suitable subjects were requested to complete a short questionnaire detailing their physical activity in the $24 \mathrm{~h}$ preceding their participation in the study. The assessing researchers were blinded to subject group allocation for the duration of the trial. Throughout the testing, the dominant leg was assessed and treated; the dominant leg was determined by asking the subjects to kick a

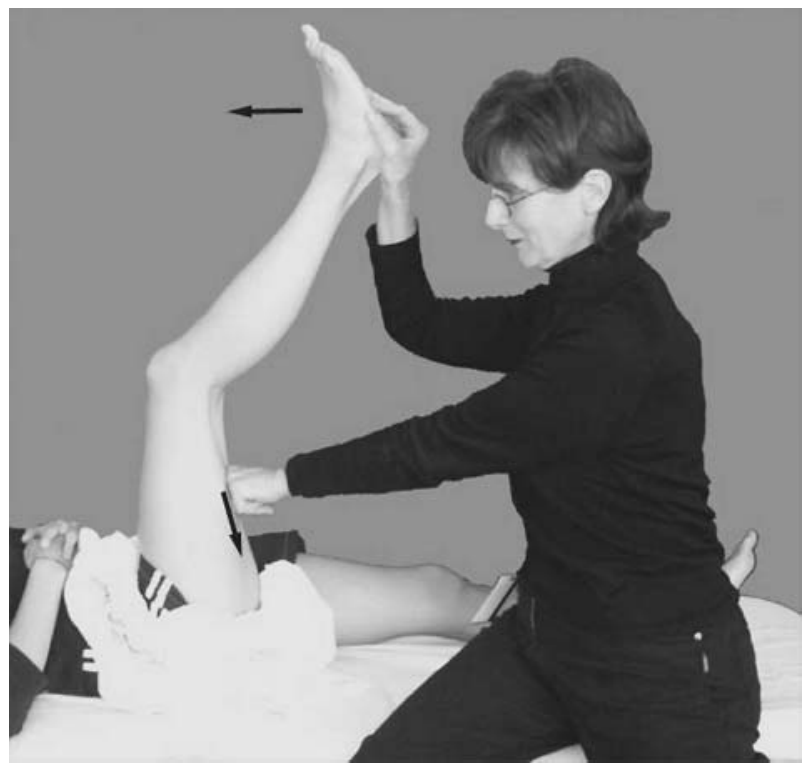

Figure 2 Using the surface area of the proximal phalanges of the fist to perform longitudinal strokes in the dynamic position. (Photograph reproduced with consent) 


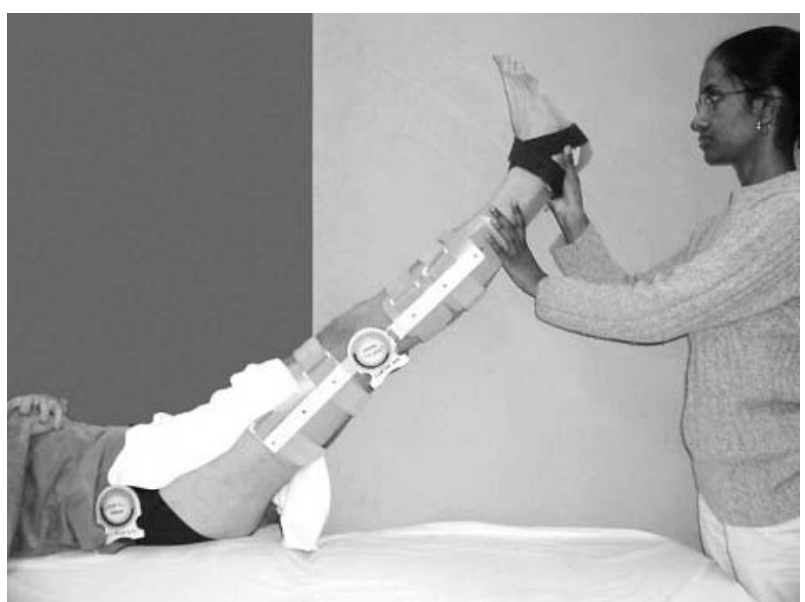

Figure 3 The SLR measurement tool. (Photograph reproduced with consent)

ball to a specific target. All instructions were standardised throughout the testing and intervention procedures.

Following pre-intervention measurements, subjects reported to the investigators responsible for the treatment aspect of the study and were randomly assigned to one of the three protocols (control, classic, or dynamic intervention) using a random numbers table. These investigators were blinded to both pre- and post-intervention HFA measurements. There was minimal delay in post-intervention as testing was commenced within $90 \mathrm{~s}$ of the completion of the intervention.

\section{Measurement of hamstring flexibility}

Hamstring flexibility was determined by measuring SLR using the same instrumentation and method previously described by Hall et al. ${ }^{23}$ In brief, the HFA measurement tool involved the application of a knee extension brace and an ankle foot orthosis to maintain the knee in full extension and the ankle in neutral plantar grade. A belt was placed around the subject at the level of the anterior superior iliac spine to provide a site of attachment for the pelvic inclinometer (Chattanooga Group Baseline, Hixson, TN, USA). A second inclinometer was attached to the brace at the level of the lateral axis of the knee joint. The two inclinometers were calibrated in the starting position and then used to measure posterior pelvic rotation and the total range of SLR. The hip flexion component of the overall measurement was considered to most closely reflect hamstring flexibility, so for the purposes of this study, HFA was defined as the difference between the total range of SLR and the range of pelvic rotation. To further standardise the position, all subjects were positioned supine without a pillow and maintained a neutral cervical spine posture throughout the measurement procedure.

To ensure consistency in all measurements, the same investigator lifted the leg passively, stopping at the point where the subject reported the onset of perceived hamstring tightness. To overcome initial hamstring tightness, the test movement pattern of an SLR was repeated four times prior to any measurements being recorded. ${ }^{24}$ During testing, the SLR was repeated three times and the pelvic and knee inclinometer readings were recorded. The HFA was calculated for each of the three trials and a mean pre-HFA value was obtained for each subject on each occasion of testing (fig 3 ).

The SLR measurement tool has been shown to be reliable in a previous study. ${ }^{23}$ These authors found high intra-tester reliability for measurement of SLR (intraclass correlation (ICC) 0.99 ) and pelvic rotation (ICC 0.94). In the current study, reliability testing using the same tool was conducted on 15 subjects prior to the main study confirming comparable intra-tester reliability for the HFA measurement $\left(\operatorname{ICC}_{(3,1)}\right.$ 0.95 ) and a standard error of measurement of $1.8^{\circ}$.

\section{Statistical analysis}

Statistical analysis was performed using SPSS version 11.0. Comparability of groups at baseline was checked using oneway ANOVA. A one-way ANCOVA was used to determine whether the change in HFA differed between groups. Change in HFA was calculated as the difference between the pre- and post-intervention HFA measurements. The covariate was the pre-intervention HFA. The least significant difference (LSD) procedure, which is suitable for pairwise comparisons where there are three groups, was used for post-hoc comparisons. ${ }^{25}$ With the LSD procedure, $\alpha$ is not adjusted for pairwise comparisons. For all analyses, $\alpha$ was designated as 0.05 .

\section{RESULTS}

Subjects $(n=45)$ completed all the dynamic phases in the experimental protocol . Baseline data analysis indicated that the subjects in the three conditions were comparable with respect to age, pre-test activity level, and pre-HFA values (table 1).

The mean change in HFA (degrees) for each condition is shown in table 2 . There was a significant difference between the three conditions $\left(F_{2,41}=5.03, \mathrm{p}=0.01\right)$. The pre-intervention HFA was not significantly associated with the change in HFA $(p=0.2)$. Post-hoc pairwise comparisons revealed that the change in HFA in the dynamic intervention group was significantly greater than the change in either the control or classic intervention groups (table 2). However, the classic intervention group did not differ significantly from the control group $(\mathrm{p}=0.26)$.

\section{DISCUSSION}

Our study found that subjects who received dynamic STM achieved significantly greater increases in hamstring flexibility than the control and classic STM groups. In two recent studies, hamstring massage was applied using typical Swedish massage protocols for $15-20 \mathrm{~min}$. The outcomes of these studies demonstrated that massaging the hamstring showed no improvement in the sit and reach test ${ }^{26}$ and no measurable physiological effect after a 20 min post-recovery leg massage. ${ }^{27}$ It could be suggested that these studies used

Table 1 Summary values for the subjects in the three conditions

\begin{tabular}{|c|c|c|c|c|}
\hline & Control $(n=15)$ & Classic intervention $(n=15)$ & Dynamic intervention $(n=15)$ & $p$ value \\
\hline Age, years (SD) & $22.0(3.3)$ & $24.5(4.8)$ & $24.5(5.3)$ & 0.22 \\
\hline \multirow[t]{2}{*}{$\begin{array}{l}\text { Activity level within } 24 \mathrm{~h} \text { prior } \\
\text { to study, number of subjects }\end{array}$} & $11<1 \mathrm{~h}$ & $11<1 \mathrm{~h}$ & $8<1 h$ & 0.28 \\
\hline & $4 \geqslant 1 \mathrm{~h}$ & $4 \geqslant 1 h$ & $7 \geqslant 1 \mathrm{~h}$ & \\
\hline Pre-HFA, degrees (SD) & $30.7(6.8)$ & $35.3(7.1)$ & $35.0(6.2)$ & 0.12 \\
\hline Post-HFA, degrees (SD) & $30.7(7.6)$ & $36.5(5.2)$ & $39.7(8.6)$ & NA \\
\hline
\end{tabular}


generalised Swedish massage techniques that were performed with the muscles in a neutral position and that the techniques were too non-specific to the areas of muscle tightness. Our study demonstrated that a significant increase in hamstring length could be achieved by identifying a specific area of hamstring tightness and targeting treatment to this area using dynamic techniques. Furthermore, these specific changes occurred within an 8 min time frame which is more time efficient compared with the previous two studies where treatment was of a substantially longer duration. ${ }^{26} 27$

The classic component of dynamic STM exactly replicated the classic protocol and was comprised of the traditional Swedish soft tissue techniques used in previous studies. ${ }^{14-17} 19$ This suggests that the unique features of dynamic STM resulted in the significant difference in HFA measurements between this intervention and the control and the classic STM groups. Most of the current literature describes general techniques applied to non-specific areas delivered when muscles were placed in a shortened, resting, or neutral position $^{14}{ }^{16-19}$ Unlike these generalised techniques, dynamic STM is a specific structured technique in which the therapist identifies a target area of muscle tightness and focuses the treatment on that specific area whilst moving it longitudinally under different muscle contraction parameters.

In the dynamic STM component, the hamstring muscle group receives progressive dynamic techniques that work in synchrony as the muscle moves to the end ROM. The final technique eccentrically works the muscle at its functional length with the result that hamstring flexibility is optimised.

Since reduced muscle flexibility has previously been linked with an increased risk of injury, ${ }^{528}$ this finding may be of clinical relevance. At present, there is a lack of literature demonstrating effective methods of increasing flexibility and the purported benefits of stretching programs remain largely unproven or inconclusive. ${ }^{10}$ Similarly, despite the common assumption that manual techniques enhance tissue extensibility, most of the literature evaluating the effectiveness of soft tissue techniques has not demonstrated positive outcomes. ${ }^{13} 1718$

Some limitations of the study have been identified which may have affected the magnitude of the change in HFA in the dynamic intervention group. An important limitation was the use of a specific sample without a history of hamstring pathology. The selection of a heterogeneous group without excessive hamstring tightness may be a factor contributing to the low mean increase in HFA. However, despite this limitation, a statistically significant difference in response to intervention was found, which suggests that dynamic STM has advantages over classic STM.

A second possible explanation for the magnitude of the change in HFA following dynamic STM intervention is that muscle tightness is difficult to quantify. HFA was selected as a means of measuring the effect of dynamic STM; however, tissue compliance and resistance to motion may be more suitable outcome measures for evaluating this technique. It has been documented that hamstring muscle injuries

Table 2 Mean (SD) degree change in HFA measures

\begin{tabular}{|c|c|c|c|}
\hline Group & n & $\begin{array}{l}\text { Degree change in } \\
\text { HFA, mean (SD) }\end{array}$ & $\begin{array}{l}\mathrm{p} \text { value for } \\
\text { comparison with } \\
\text { dynamic intervention }\end{array}$ \\
\hline Control & 15 & $-0.04(4.8)$ & 0.003 \\
\hline $\begin{array}{l}\text { Classic } \\
\text { intervention }\end{array}$ & 15 & $1.3(3.8)$ & 0.049 \\
\hline $\begin{array}{l}\text { Dynamic } \\
\text { intervention }\end{array}$ & 15 & $4.7(4.8)$ & NA \\
\hline
\end{tabular}

What is already known on this topic

It is widely believed amongst athletes, coaches, and therapists that massage is an effective treatment modality for increasing flexibility, although recent studies have shown that a $20 \mathrm{~min}$ massage has no effect.

\section{What this study adds}

Our study shows that dynamic soft tissue mobilisation had an immediate effect on hamstring muscle length, and hence flexibility. These results are unique, have not been previously reported in the literature, and may be worthy of clinical consideration.

typically occur when the muscle is well within its limits of ROM. ${ }^{29}$ Reducing tissue tension within the functional ROM of each athlete is the primary goal of dynamic STM. Therefore, a test of muscle stiffness or compliance through range may be a more effective way to evaluate dynamic STM.

The dynamic STM was 3 min longer in duration than the other two interventions. The extra $3 \mathrm{~min}$ of massage may have influenced the results. However, this is considered unlikely to explain the observed differences as a previous study found that 15 min of Swedish massage did not improved hamstring length measured using the sit and reach test. ${ }^{26} 27$ This study was not designed to explore the relative contribution of individual components of the dynamic STM model to the benefits gained.

To explore the efficacy of dynamic STM, further research is required to focus on sports specific athletes, such as cyclists and football and hockey players, who are frequently troubled by hamstring muscle tightness. This study demonstrated a significant short term effect on hamstring muscle flexibility, but longer term effects need to be established over different time frames. Compliance or resistance to motion may assist in determining the true effect of dynamic STM. Anecdotally, massage is often viewed as a time consuming and nonspecific treatment option in clinical practice. The improvement in HFA in response to dynamic STM was achieved with very little additional treatment time compared to classic STM. The positive outcome of this study is consistent with anecdotal reports on the benefits of dynamic STM in the clinical setting.

\section{ACKNOWLEDGEMENTS}

A special thank you to the subjects who gave of their time willingly and without whose generosity this study would not have been possible.

\section{Authors' affiliations}

D Hopper, K Briffa, Curtin University of Technology, Shenton Park, WA 6008, Australia

S Deacon, S Das, A Jain, D Riddell, T Hall, Private Practice, Perth, Australia

Competing interests: none declared

Consent was obtained for the publication of figures in this article

\section{REFERENCES}

1 Hutson M. Sports injuries: recognition and management, 2nd ed. Oxford: Oxford University Press, 1996.

2 Hartig DE, Henderson JM. Increasing flexibility decreases lower extremity overuse injuries in military basic trainees. Am J Sports Med 1999;27:173-6. 
3 Gleim GW, McHugh MP. Flexibility and its effects on sports injury and performance. Sports Med 1997;24:289-99.

4 Pope R, Herbert R, Kirwan J, et al. A randomized trial of pre-exercise stretching for prevention of lower limb injury. Med Sci Sports Exer 2000;32:271-7.

5 Witvrouw E, Lysens R, Bellemans J, et al. Intrinsic risk factors for the development of anterior knee pain in an athletic population: a two year prospective study. Am J Sports Med 2000;28:480-9.

6 Witvrouw E, Bellemans J, Lysens R, et al. Intrinsic risk factors for the development of patellar tendinitis in the athletic population. Am J Sports Med 2001;29:190-5.

7 McHugh M, Connolly D, Eston R, et al. The role of passive stiffness in symptoms of exercise-induced muscle damage. Am J Sports Med 1999:27:594-9.

8 Brukner P, Khan K. Clinical sports medicine, 2nd ed. Roseville, NSW: McGraw-Hill, 2001.

9 Bandy W, Irion J, Briggler M. The effect of static and dynamic range of motion training on the flexibility of the hamstring muscles. J Orthop Sports Phys Ther 1998;27:295-300.

10 Herbert R, Gabriel M. Effects of stretching before and after exercising on muscle soreness and risk of injury: systematic review. Br Med J 2002;325:468-78.

11 Shrier I. Stretching before exercise does not reduce the risk of local muscle injury: a critical review of the clinical and basic science literature. Clin J Sport Med 1999;9:221-7.

12 Hemmings B. Physiological, psychological and performance effects of massage therapy in sport: a review of the literature. Phys Ther Sport 2001:2:165-70

13 Tiidus P. Manual massage and recovery of muscle function following exercise: a literature review. J Orthop Sports Phys Ther 1997;25:107-12.

14 Tiidus $\mathbf{P}$, Shoemaker J. Effleurage massage, muscle blood flow and long-term post-exercise strength recovery. Int J Sports Med 1995;16:478-83.

15 Ernst $E$. Does post-exercise massage treatment reduce delayed onset muscle soreness? A systematic review. Br J Sports Med 1998;32:212-4.

16 Smith L, Keating M, Holbert D, et al. The effects of athletic massage on delayed onset muscle soreness, creatine kinase, and neutrophil count: a preliminary report. J Orthop Sports Phys Ther 1994;19:93-8.

17 Hilbert J, Sforzo G, Swensen T. The effects of massage on delayed onset muscle soreness. Br J Sports Med 2003;37:72-9.

18 Cafarelli E, Sim J, Carolan B, et al. Vibratory massage and short-term recovery from muscular fatigue. Int J Sports Med 1990;11:474-8.

19 Rodenburg J, Steenbeek D, Schiereck P, et al. Warm-up, stretching and massage diminish harmful effects of eccentric exercise. Int J Sports Med 1994;15:414-9.

20 Hopper D. A new dynamic deep muscle tissue model (DDMT). In: Proceedings of the Annual Scientific Conference in Sports Medicine. Canberra, ACT, 1991.

21 Hunter G. Specific soft tissue mobilization in the management of soft tissue dysfunction. Man Ther 1998;3:2-11.

22 Goats G. Massage - the scientific basis of an ancient art: part 2. Physiological and therapeutic effects. Br J Sports Med 1994;28:149-52.

23 Hall T, Cacho A, McNee C, et al. Effects of the Mulligan traction straight leg raise technique on range of movement. J Man Manipulative Ther $2001 ; 9: 128-33$.

24 Taylor D, Dalton J, Seaber A, et al. Viscoelastic properties of muscle-tendon units: the biomechanical effects of stretching. Am J Sports Med 1990;18:300-9.

25 Green SB, Slakind NJ, Akey TM. Using SPSS for Windows: analyzing and understanding data, 2nd ed. Upper Saddle River, NJ: Prentice Hall, 2000.

26 Barlow A, Clarke R, Johnson N, et al. Effect of massage of the hamstring muscle group on performance of the sit and reach test. Phys Ther Sport 2004;38:349-51.

27 Robertson A, Watt JM, Galloway SDR. Effects of leg massage on recovery from high intensity cycling exercise. Phys Ther Sport 2004:38:173-6.

28 Witvrouw E, Danneels L, Asselman P, et al. Muscle flexibility as a risk factor for developing muscle injuries in male professional soccer players: a prospective study. Am J Sports Med 2003;31:41-7.

29 Wilson G, Wood G, Elliott B. The relationship between stiffness of the musculature and static flexibility. Int J Sports Med 1991;12:403-7.
COMMENTARY

Dynamic soft tissue mobilization (STM) follows a relatively recent development in manual therapy techniques ${ }^{12}$ in that it combines, with the therapist delivered manual treatment, a number of different features, such as: passive joint and soft tissue positioning and movements; and/or active movements involving either concentric or eccentric muscle activity. Invariably linked with, and preceding, these manual therapy techniques is a precise and careful manual examination that relies on the therapist's perception of the tissue's compliance and response to manual provocation, including any induced muscle reactivity. This examination then guides the manual therapy treatment.

There is a consistent theme throughout this paper that asserts that a distinguishing feature of dynamic STM is the targeting of specific areas of tight hamstring muscles. It is important to recognise that, as an integral part of its implementation, the dynamic STM technique involves not only the specific targeting of areas of tightness in muscles but also end-range procedures (passive, concentric then eccentric contractions). It is difficult to decide from this study the relative contribution or significance of the manual therapy targeting of the specific area of tightness within the muscle. While the authors state that it was not their intention to evaluate this, I believe that healthcare practitioners would be well served by information that either supports or refutes the necessity to identify specific areas of tightness within muscles. For example, if the end-range procedures performed without the specific manual therapy are as effective in increasing hip flexion angle (HFA), the patient could perform those procedures by themselves under prescription and regular monitoring by the healthcare practitioner. This would also allow the patient to perform these techniques outside the clinic as a self-treatment, which is another consistent feature of modern manual therapy techniques that is often required as the initial effect of manual therapy is usually short lived.

Notwithstanding the above comments, this study provides evidence of an initial effect of dynamic STM beyond that of massage performed in a neutral aligned and passive lower limb. This is valuable information for healthcare practitioners who use soft tissue manipulation that combines manual therapy of the soft tissues with various limb postures and movements as well as states of muscle contraction.

Bill (Guglielmo) Vicenzino Department of Physiotherapy, University of Queensland, Queensland, Australia; b.vicenzino@shrs.uq.edu.au

\section{REFERENCES}

1 Edwards BC. Manual of combined movements, 2nd ed. Oxford: ButterworthHeinemann, 1999:160.

2 Mulligan BR. Manual therapy - "NAGS", "SNAGS", "MWMS" etc, 4th ed. Wellington: Plane View Services, 1999:142. 